\title{
ON CO-GROUPS IN THE CATEGORY OF GRADED ALGEBRAS $\left({ }^{(}\right)$
}

\author{
BY \\ ISRAEL BERSTEIN
}

1. Introduction. Let $\mathscr{C}$ be any category. An object $B \in \mathscr{C}$ is called a group in $\mathscr{C}$ (or, more strictly, is given a structure of a group in $\mathscr{C}$ ) if for any $A \in \mathscr{C}$ the set $\operatorname{Hom}(A, B)$ is given a group structure, such that for any $f: A^{\prime} \rightarrow A^{\prime \prime}$ the induced map $f^{*}: \operatorname{Hom}\left(A^{\prime \prime}, B\right) \rightarrow \operatorname{Hom}\left(A^{\prime}, B\right)$ is a homomorphism. Of course not every object of $\mathscr{C}$ can be so structured. Dually, an object $A \in \mathscr{C}$ is called a co-group if it is a group in the dual category, i.e., for each $B \in \mathscr{C}, \operatorname{Hom}(A, B)$ is given a group structure, such that for any $g: B^{\prime} \rightarrow B^{\prime \prime}$ the induced map $g_{*}: \operatorname{Hom}\left(A, B^{\prime}\right) \rightarrow \operatorname{Hom}\left(A, B^{\prime \prime}\right)$ is a homomorphism. The importance of groups and co-groups in different categories has been emphasized in [2].

In the category $\mathscr{T}_{H}$ of based topological spaces and homotopy classes of maps, the co-groups are the so-called $H^{\prime}$-spaces $\left({ }^{2}\right)$ (e.g. any suspension is such an $H^{\prime}$-space). The following facts are well known.

(1) The fundamental group functor $\pi_{1}$ (for spaces with "nice" base point) preserves free products (in $\mathscr{T}_{H}$ the free product is the wedge of spaces $X \bigvee Y$ and $\left.\pi_{1}(X \bigvee Y)=\pi_{1}(X) * \pi_{1}(Y)\right)$.

(2) The fundamental group of an $H^{\prime}$-space is free.

Now, in a category with zero maps and free products a more direct and workable definition of co-groups may be given (see Definition 2.1). It follows from it that if a functor between two categories preserves free products, it also preserves co-groups. Therefore, the "categorical" explanation of (2) in view of (1) follows from a result of D. M. Kan [4], who has proved that a co-group in the category $\mathscr{G}$ of groups is always a free group. Kan's theorem shows that the co-group structure of $G \in \mathscr{G}$ completely determines a set of free generators of $G$ (the so called "primitive" elements).

On the other hand it is a fact that

$\left(1^{\prime}\right)$ The Pontrjagin algebra of the loop space $H_{*}(\Omega ; K)$, where $K$ is a field, is another functor which preserves free products (since no published proof of this probably well-known result is known to the author, we shall give one in \$3). Moreover,

(2') The Bott-Samelson theorem [1] states that $H_{*}(\Omega \Sigma X ; K)$ ( $\Sigma$ being the suspension functor) is a free algebra (generated by $H_{*}(X ; K)$ ).

The aim of this paper is to prove some purely algebraic results which

Received by the editors December 31, 1963.

(1) Supported by National Science Foundation grant NSF G-15984.

$\left.{ }^{2}\right)$ Or $G^{\prime}$-spaces in more recent terminology. 
will enable us to derive $\left(2^{\prime}\right)$ from $\left(1^{\prime}\right)$ in the same way as the result of Kan gives the possibility of deriving (2) from (1).

Kan's theorem quoted above has been later extended in a slightly weakened form to the more general case when no associativity is required [3]. Certain of our results will also hold for "co-multiplicative objects" ${ }^{(3)}$ rather than for co-groups. The definition of a co-multiplicative object (c.-m. object) may be obtained from the above definition of a co-group by replacing the words "group structure". by "structure of a multiplicative system with two-sided unit." Again a more convenient equivalent definition will be given in $\$ 2$.

Let $\mathscr{A}$ be the category of connected graded associative algebras over a commutative ring $\Lambda$ and let $\mathscr{A}_{H}$ be the "primitive" [2, Part II] category of c.-m. objects in $\mathscr{A}$. Our first main result is

THEOREM 1.1. There exists a functor $N: \mathscr{A}_{H} \rightarrow \mathscr{M}$ (where $\mathscr{M}$ is the category of positively graded modules over $\Lambda)$ such that

(i) $N(A)$ is a submodule of $A\left(A \in \mathscr{X}_{H}\right)$ called the module of normed elements and

(ii) as an algebra, $A$ is freely generated by $N(A)$, i.e., $A=T(N(A))$, where $T(N)$ is the tensor algebra of $N$; in particular, any object of $\mathscr{X}_{H}$ is a free algebra.

In the case of co-groups we have a more precise statement. Let $\mathscr{A}_{G}$ be the category of co-groups in $\mathscr{A}$ and let $\mathscr{C}$ be the category of connected graded co-associative co-algebras (with co-unit) over $\Lambda$.

Theorem 1.2. There exist two covariant functors $S: \mathscr{A}_{G} \rightarrow \mathscr{C}$ and $T_{G}$ : $\mathscr{C} \rightarrow \mathscr{A}_{G}$ such that

(i) both compositions $T_{G} S$ and $S T_{G}$ are equal to the identity,

(ii) $S(A)$ for $A \in \mathscr{A}_{G}$ is precisely the submodule of normed elements of $A$, and

(iii) as an algebra, $T_{G}(S(A))$ is the free (tensor) algebra generated by $S(A)$. Thus, in particular, $\mathscr{A}_{G}$ and $\mathscr{C}$ are category isomorphic.

The strength of Theorem 1.2 resides in the fact that the co-algebra structure of $S(A)$ completely determines the co-group structure of $A$ and conversely. The precise way in which this is done will be described in $\$ 2$. Since the elements of $S(A)$ are "semi-primitive" in a certain sense, Theorem 1.2 is the analogue for algebras of Kan's theorem [4].

Let now $\Lambda=K$ be a field and suppose that $\mathscr{A}$ is the category of connected graded algebras over $K$, of finite type. Let $C^{*}$ be the dual algebra of the co-algebra $C$.

(3) $\mathrm{H}^{\prime}$-objects in the terminology of [2]. 
Corollary 1.3. The contravariant functor $S^{*}: \mathscr{A}_{G} \rightarrow \mathscr{A}$ defined by $S^{*}(A)=(S(A))^{*}$ establishes an anti-isomorphism between the two categories.

Let $Y$ be a topological space with finitely generated homology, and let $\Sigma Y$ be the (reduced) suspension of $Y$. It is well known that $\Sigma Y$ is a cogroup in $\mathscr{T}_{H}$; as shown in the proof of Corollary 3.3 this co-group structure induces a canonical co-group structure in the Pontrjagin algebra of the loop space $\Omega \Sigma Y$ of $\Sigma Y$ (provided we use a field $K$ of coefficients or no torsion is present).

Corollary 1.4. $S^{*}\left(H_{*}(\Omega \Sigma Y, K)\right)=H^{*}(Y, K)$, the latter being the cohomology algebra of $Y$.

$\$ 2$ contains the main definitions used in the paper and also the proof of the results stated above (modulo two key lemmas, whose proof is relegated to the completely technical $\$ 4)$. $\$ 3$ is devoted to the topological implication of our results.

2. Definitions and proof of the theorems. A category $\mathscr{C}$ is said to possess zero maps (morphisms) if for each $A, B, \in \mathscr{C}$, the set $\operatorname{Hom}(A, B)$ contains a distinguished element 0 satisfying $0 f=0, g 0=0$ for all $f \in \operatorname{Hom}\left(A^{\prime}, A\right)$, $g \in \operatorname{Hom}\left(B, B^{\prime}\right)$.

We shall denote by 1 both the identity functor (in any category) and the identity map (of any object).

Let $A_{1}, A_{2} \in \mathscr{C}$. A free (or inverse) product [2] of the objects $A_{1}, A_{2}$ is an object $Q$ and a pair of maps $i_{j}: A_{j} \rightarrow Q, \quad j=1,2$, such that for any $X$ $\in \mathscr{C}$ and any two maps $f_{j}: A_{j} \rightarrow X, j=1,2$, there exists a unique map $f$ : $Q \rightarrow X$ with $f i_{j}=f_{j}$. We write $f=\left\langle f_{1}, f_{2}\right\rangle$; thus $\left\langle f_{1}, f_{2}\right\rangle i_{j}=f_{j}$. The free product, if it exists, is unique up to an equivalence. We will denote by $A_{1} * A_{2}$ an arbitrary respresentative of the class of equivalent free products of $A_{1}, A_{2}$. The free product is associative in the obvious sense. If $g_{j}$ : $A_{j}^{\prime} \rightarrow A_{j}, j=1,2$, we write $g_{1} * g_{2}$ for the $\operatorname{map}\left\langle i_{1} g_{1}, i_{2} g_{2}\right\rangle: A_{1}^{\prime} * A_{2}^{\prime} \rightarrow A_{1} * A_{2}$.

From now on $\mathscr{C}$ will be a category with zero maps and free products. The following definitions are equivalent to the ones given in Introduction [2, Part I, Theorem 4.6].

Definition 2.1. A co-multiplication on $A \in \mathscr{C}$ is a map

$$
\Phi: A \rightarrow A * A
$$

such that $\langle 1,0\rangle \Phi=\langle 0,1\rangle \Phi=1: A \rightarrow A$. The pair $(A, \Phi)$ will be then called a co-multiplicative object (c.-m. object). A co-multiplication $\Phi$ is associative if

$$
(1 * \Phi) \Phi=(\Phi * 1) \Phi: A \rightarrow A * A * A .
$$

$\Phi$ has an inverse $\nu: A \rightarrow A$ if $\langle\nu, 1\rangle \Phi=\langle 1, \nu\rangle \Phi=0$. If $\Phi$ is associative and has an inverse, then $(A, \Phi)$ is called a co-group. 
If $\left(A^{\prime}, \Phi^{\prime}\right)$ and $\left(A^{\prime \prime}, \Phi^{\prime \prime}\right)$ are c.-m. objects in $\mathscr{C}$, a map $f: A^{\prime} \rightarrow A^{\prime \prime}$ is a homomorphism if $\Phi^{\prime \prime} f=(f * f) \Phi^{\prime}$. The category of all c.-m. objects in $\mathscr{C}$ and their homomorphisms will be denoted by $\mathscr{L}_{H}$; the corresponding category of co-groups will be denoted by $\mathscr{L}_{G}$; obviously $\mathscr{C}_{G} \subset \mathscr{L}_{H}$.

EXAmple 2.2. In the category $\mathscr{T}_{H}$ of based topological spaces, the free product is the wedge $X \bigvee Y$ (union with a common base-point). $A$ comultiplication $\Phi$ in $\mathscr{T}_{H}$ is a map $\Phi: X \rightarrow X \bigvee X$, such that $r_{1} \Phi \sim r_{2} \Phi \sim 1$ : $X \rightarrow X$, where $r_{1}, r_{2}: X \bigvee X \rightarrow X$ are the retractions onto the two factors.

Let now $\Lambda$ be a fixed commutative ring and $\mathscr{A}$ the category of connected associative graded algebras over $\Lambda$. Any $A \in \mathscr{A}$ is provided with an augmentation $\epsilon: A \rightarrow \Lambda$ which maps $A^{0}$ (the module of elements of degree 0 ) isomorphically onto $\Lambda$ and which vanishes on elements of positive degree; we denote $\operatorname{Ker} \epsilon$ by $\bar{A}$. Any map $f$ in $\mathscr{A}$ satisfies $\epsilon=\epsilon$; the zero map 0: $A \rightarrow B, A, B \in \mathscr{A}$ is characterized by $0(\bar{A})=0$.

If $N$ is a positively graded $\Lambda$-module we denote by $T(N)$ the tensor algebra of $N$.

In order to be able to describe in a convenient way the free product in $\mathscr{A}$ we shall introduce first some notations which will be used throughout the paper.

Let $\alpha, \beta$ be any two symbols. $A$ sequence of the form $(\alpha, \beta, \alpha, \beta, \ldots)$ or $(\beta, \alpha, \beta, \alpha, \cdots)$ will be called alternating. There are exactly two alternating sequences of length $n$ : the first starting with $\alpha$ and the second starting with $\beta$. The set of all alternating sequences will be denoted by $\mathscr{R}=\mathscr{R}(\alpha, \beta)$. If $I=\left(i_{1}, \cdots, i_{n}\right), J=\left(j_{1}, \cdots, j_{p}\right)$ are two alternating sequences, define

$$
\begin{array}{ll}
I \bigvee J=\left(i_{1}, \cdots, i_{n}, j_{1}, \cdots, j_{p}\right) & \text { if } i_{n} \neq j_{1}, \\
I \bigvee J=\left(i_{1}, \cdots, i_{n}, j_{2}, \cdots, j_{p}\right) & \text { if } i_{n}=j_{1} .
\end{array}
$$

If $I \in \mathscr{R}(1,2)$ set

$$
\overline{A_{I}}=\overline{A_{i_{1}}} \otimes \cdots \otimes \overline{A_{i_{n}}}, \quad I=\left(i_{1}, \cdots, i_{n}\right) .
$$

Define, for $A_{1}, A_{2} \in \mathscr{A}$

$$
A_{1} * A_{2}=\Lambda+\Sigma \overline{A_{I}}, \quad I \in \mathscr{R}(1,2) .
$$

$A_{1} * A_{2}$ has the obvious grading and augmentation; it can be converted into an algebra by means of the multiplication

$$
\mu_{I J}: \overline{A_{I}} \otimes \overline{A_{J}} \rightarrow \overline{A_{I} \vee J},
$$

where $\mu_{I J}$ is the canonical isomorphism if $i_{n} \neq j_{1}$ and is induced by the multiplication $\overline{A_{i_{n}}} \otimes \overline{A_{j_{1}}} \rightarrow \overline{A_{i_{n}}}$ and the identity on the other factors if $i_{n}$ $=j_{1}$. If we define $i_{k}: A_{k} \rightarrow A_{1} * A_{2}, k=1,2$, as the obvious imbeddings, all the axioms of a free product are satisfied. For instance, if $f_{k}: A_{k} \rightarrow B$, $k=1,2$, then $\left\langle f_{1}, f_{2}\right\rangle \mid \overline{A_{I}}, I \in \mathscr{R}$ is defined as being the composition 


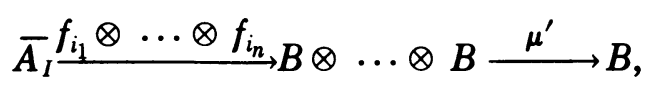

where $\mu^{\prime}$ is the multiplication in $B$. In particular,

$$
\begin{aligned}
& \langle 1,0\rangle \mid \Lambda+\overline{A_{1}}: \Lambda+\overline{A_{1}} \approx A_{1}, \\
& \langle 0,1\rangle \mid \Lambda+\overline{A_{2}}: \Lambda+\overline{A_{2}} \approx A_{2},
\end{aligned}
$$

all the other summands being mapped onto zero.

We now adopt the following convention: if $a \in A$ we use a prime sign $\left(a^{\prime}\right)$ in order to indicate that $a$ belongs to the first factor of $A * A$ and a double prime sign $\left(a^{\prime \prime}\right)$ in order to indicate that $a$ belongs to the second factor. The following is a consequence of Definition 2.1 and of (2.4).

Proposition 2.3. A co-multiplication on $A \in \mathscr{A}$ is a map $\Phi: A \rightarrow A * A$ satisfying

$$
\Phi(a)=a^{\prime}+a^{\prime \prime}+b, \quad b \in \Sigma A_{I}, \quad I \in R\left({ }^{\prime},,^{\prime \prime}\right), \quad|I| \geqq 2
$$

(where $|I|$ is the length of the sequence $I$ ) for all $a \in A$ (b depends on $a$ ).

For $A_{j} \in \mathscr{A}, j=1,2$, define $k_{j}: A_{j} \rightarrow A_{1} \otimes A_{2}$ by $k_{1}\left(a_{1}\right)=a_{1} \otimes 1, k_{2}\left(a_{2}\right)$ $=1 \otimes a_{2}$. Then

$$
\omega=\left\langle k_{1}, k_{2}\right\rangle: A_{1} * A_{2} \rightarrow A_{1} \otimes A_{2}
$$

maps the free product of algebras onto their tensor product. If $\Phi: A \rightarrow A * A$ is a co-multiplication, then the "diagonal map" $\Delta=\omega \Phi$ converts $A$ to a Hopf algebra called the underlying Hopf algebra of $(A, \Phi)$. On the other hand if $\mu:(A * A) \otimes(A * A) \rightarrow A * A$ is the multiplication in $A * A$ and $i_{j}: A \rightarrow A * A, j=1,2$, are the two imbeddings, then

$$
\rho=\mu\left(i_{1} \otimes i_{2}\right) \Delta=\mu\left(i_{1} \otimes i_{2}\right) \omega \Phi: A \rightarrow A * A
$$

is a map of graded modules.

THEOREM 1.1 is an immediate consequence of the following

Lema 2.4. For any c.-m. object $(A, \Phi), A \in \mathscr{A}$, we can define in a canonical way a submodule $N \subset A$ such that $A$ is freely generated by $N$, i.e., $A=T(N)$; if $f:\left(A_{1}, \Phi_{1}\right) \rightarrow\left(A_{2}, \Phi_{2}\right)$ is a homomorphism then $f\left(N_{1}\right) \subset N_{2}$.

The elements $a \in N$ are called normed. The construction of normed elements and the proof of Lemma 2.4 will be obtained in $\$ 4$ by induction on the degree.

Let $S=\operatorname{Ker}(\Phi-\rho)$, where $(A, \Phi)$ is a c.-m. object in $\mathscr{A}$ and $\rho$ is given by (2.5). Since

$$
\Delta(a)=a \otimes 1+1 \otimes a+\Sigma a_{i} \otimes a_{j}, \quad a \in A
$$

for any $a \in S$, we have

$$
\Phi(a)=\rho(a)=\mu\left(i_{1} \otimes i_{2}\right) \Delta(a)=a^{\prime}+a^{\prime \prime}+\Sigma a_{i}^{\prime} a_{j}^{\prime \prime} .
$$


Formula (2.6') suggests calling the elements of $S$ semi-primitive.

LEMma 2.5. If $\Phi$ is associative then

(i) all normed elements are semi-primitive, i.e., $N=S$, and

(ii) $S$ is a sub-co-algebra of the underlying Hopf algebra of $(A, \Phi)$, i.e., $a \in S$ implies that in (2.6) $a_{i}, a_{j} \in S$.

This lemma will also be proved in $\$ 4$.

We are now able to prove Theorem 1.2.

Proof of Theorem 1.2. The functorial character of $S=S(A)$ follows from the fact that if $f:(A, \Phi) \rightarrow\left(A^{\prime}, \Phi^{\prime}\right)$ is a homomorphism of co-groups, $\Phi^{\prime} f$ $=(f * f) \Phi$ and $\rho^{\prime} f=(f * f) \rho$ and thus $f(\operatorname{Ker}(\Phi-\rho)) \subset \operatorname{Ker}\left(\Phi^{\prime}-\rho^{\prime}\right)$. Lemma 2.5 together with Theorem 1.1 show us that as an algebra, $A=T(S(A))$. Given an arbitrary co-algebra $C \in \mathscr{C}$ with diagonal map $\bar{\Delta}: C \rightarrow C \otimes C$, let $\alpha: C \rightarrow T(C)$ be the canonical inclusion; define

$$
\Delta=(\alpha \otimes \alpha) \bar{\Delta}: C \rightarrow T(C) \otimes T(C)
$$

and

$$
\rho=\mu\left(i_{1} \otimes i_{2}\right) \Delta: C \rightarrow T(C) * T(C) .
$$

By universality of the tensor algebra $T(C), \rho$ can be extended to a unique map of algebras

$$
\Phi: T(C) \rightarrow T(C) * T(C) .
$$

One easily checks that (a) $\Phi$ is co-multiplication and (b) $T_{G}(C)=(T(C), \Phi)$ is a co-group in $\mathscr{A}$ if and only if $C$ is co-associative. Notice that the canonical anti-automorphism $\nu$. of the underlying Hopf algebra of $T_{G}(C)$ serves as an inverse for $T_{G}(C)$; also, that the underlying Hopf algebra of $T_{G}(C)$ is co-commutative if and only if $C$ was co-commutative.

A co-multiplication

$$
\Phi: A \rightarrow A * A
$$

is commutative if we have $P \Phi=\Phi$, where $P: A * A \rightarrow A * A$ is the permutation of the two factors. Obviously, for a commutative $\Phi$ any semiprimitive $a$ is primitive, i.e., $\Phi(a)=a^{\prime}+a^{\prime \prime}$. Thus we have

CoRollary 2.6. A commutative co-group in $\mathscr{A}$ is a free algebra generated by the submodule of primitive elements. In particular, the underlying Hopf algebra of $A$ is also primitively generated.

3. Topological implications. We shall first prove the fact quoted in the Introduction under $\left(1^{\prime}\right)$.

Let $\mathscr{G}$ be the category of c.s.s. groups. $\mathscr{G}$ admits a free product $G_{1} * G_{2}$, $G_{i} \in \mathscr{G}$, where $\left(G_{1} * G_{2}\right)_{q}=G_{1 q} * G_{2 q}$ and the boundary and degeneracy operators are defined in the obvious way. We shall consider homology with coefficients in a field $K$. 
TheOREM 3.1. The homology functor $H_{*}: \mathscr{G} \rightarrow \mathscr{A}$ preserves free products, i.e., the map

$$
\nu=\left\langle i_{1^{*}}, i_{2^{*}}\right\rangle: H_{*}\left(G_{1}\right) * H_{*}\left(G_{2}\right) \rightarrow H_{*}\left(G_{1} * G_{9}\right)
$$

is an isomorphism (here $i_{j}: G_{j} \rightarrow G_{1} * G_{2}, j=1,2$, are the inclusions).

Proof. Let $A_{j}=H_{*}\left(G_{j}\right), \overline{A_{j}}=\widetilde{H}_{*}\left(G_{j}\right)$ (reduced homology), $j=1,2$. With the notations of (2.3) define

$$
D_{0} \subset D_{1} \subset \cdots \subset D_{n} \subset \cdots \subset A_{1} * A_{2}
$$

by setting

$$
\begin{aligned}
& D_{0}=K, \quad D_{1}=K+\overline{A_{1}}+\overline{A_{2}}, \\
& D_{n}=D_{1}+\Sigma \widetilde{A_{J}}, \quad|J| \leqq n, n \geqq .2 ;
\end{aligned}
$$

moreover,

$$
\text { Lim. dir. } D_{n}=A_{1} * A_{2}
$$

and

$$
D_{n} / D_{n-1}=\bar{A}_{I_{n}}+\bar{A}_{J_{n}}
$$

where $I_{n}$ and $J_{n}$ are the only alternating sequences of length $n$.

If $J=\left(j_{1}, \cdots, j_{k}\right)$, we shall use the notations $G_{J}=G_{j_{1}} \times \cdots \times G_{j_{k}}$ and $\overline{G_{J}}=G_{j_{1}} \wedge \ldots \wedge G_{j_{k}}$ (smashed product). By the Künneth formula we have

$$
\overline{A_{J}}=\widetilde{H}_{*}\left(\overline{G_{J}}\right) \text {. }
$$

On the other hand any $g \in G_{1} * G_{2}$ admits a unique representation as a reduced word $g=x_{1} \cdots x_{k}, e \neq x_{s} \in G_{j s}$. If $F_{n}$ is the subcomplex of all words of length $\leqq n$, we have

$$
H_{*}\left(G_{1} * G_{2}\right)=\operatorname{Lim} . \operatorname{dir} . H_{*}\left(F_{n}\right) ;
$$

for homology commutes with direct limits. For any sequence $J=\left(j_{1}, \cdots, j_{k}\right)$, $k \leqq n$, define a map

$$
\nu_{J}: G_{J} \rightarrow F_{n}
$$

by setting $\omega_{J}\left(x_{1}, x_{2}, \cdots, x_{k}\right)=x_{1} \cdots x_{k}$. The maps $\omega_{J}$ composed with the natural splitting $\overline{A_{J}} \rightarrow H_{*}\left(\overline{G_{J}}\right) \rightarrow H_{*}\left(G_{J}\right)$ induce maps

$$
\nu_{n}: D_{n} \rightarrow H_{*}\left(F_{n}\right)
$$

such that the diagram

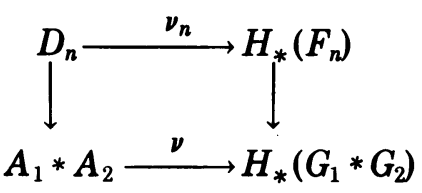


is commutative (the vertical arrows are induced by inclusion). By (3.3) and (3.6) it is enough to prove that for all $n, \nu_{n}$ is an isomorphism. This follows by repeated applications of the 5-lemma to the commutative diagram

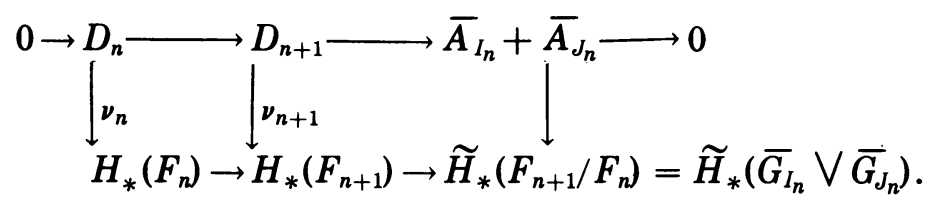

The upper exact sequence (see (3.4)) obviously splits; the third vertical arrow is an isomorphism by (3.5).

Corollary 3.2. If $X_{i}, i=1,2$, are spaces with "nice" base-point*(i.e., if * has in $X_{i}$ a neighborhood $U_{i}$ which can be deformed in $X_{i}$ into * (rel *)), then

$$
H_{*}\left(\Omega\left(X_{1} \bigvee X_{2}\right)\right) \approx H_{*}\left(\Omega X_{1}\right) * H_{*}\left(\Omega X_{2}\right) \text {. }
$$

Proof. If $S(X)$ denotes the singular complex of $X$ and the base-points are "nice", then

$$
S\left(X_{1} \vee X_{2}\right) \approx S\left(X_{1}\right) \bigvee S\left(X_{2}\right)
$$

Let $\mathscr{S}$ be the category of connected c.s.s. complexes, and let $G: \mathscr{S} \rightarrow \mathscr{G}$ be the Kan functor; according to [5] we have a natural equivalence of the functors $H * G S$ and $H_{*} \Omega$ and therefore

$$
\begin{aligned}
H_{*}\left(\Omega\left(X_{1} \bigvee X_{2}\right)\right) & =H_{*}\left(G S\left(X_{1} \bigvee X_{2}\right)\right) \\
& =H_{*}\left(G\left(S\left(X_{1}\right) \bigvee S\left(X_{2}\right)\right)\right) \\
& =H_{*}\left(G S\left(X_{1}\right) * G S\left(X_{2}\right)\right) ;
\end{aligned}
$$

it suffices now to apply Theorem 3.1.

Corollary 3.3 (BotT-Samelson). If $(X, \Phi)$ is a co-multiplicative object in $\mathscr{T}_{H}$ (i.e., $X$ is a space of normalized Lusternik-Schnirelman category $\left.\leqq 1\right)$ then $H_{*}(\Omega X)$ is a free algebra. If $\Phi$ is (iomotopy) associative, then $H_{*}(\Omega X)$ is freely generated by $\operatorname{Ker}\left[(\Omega \Phi)_{*}-\rho_{*}\right]$, where

$$
\rho=\mu\left(\Omega i_{1} \times \Omega i_{2}\right) \Delta: \Omega X \rightarrow \Omega(X \bigvee X)
$$

and $\Delta: \Omega X \rightarrow \Omega X \times \Omega X$ is the diagonal map, $i_{j}: X_{j} \rightarrow X \times X, j=1,2$, are the inclusions and $\mu$ is the multiplication of loops in $\Omega(X \vee X)$.

Proof. $\Phi$ being as in Example 2.2 it follows from Corollary 3.2 that $(\Omega \Phi)_{*}: H_{*}(\Omega X) \rightarrow H_{*}(\Omega(X \vee X))$ is a co-multiplication and thus by applying Lemmas 2.4 and 2.5 we get the result.

Proof of Corollary 1.4. In the particular case when $X$ is a suspension, i.e., $X=\Sigma Y$, we know from the original proof of the Bott-Samelson theorem, that $H_{*}(\Omega \Sigma Y)$ is freely generated by the image of $H_{*}(Y)$ un- 
der the imbedding $e: Y \rightarrow \Omega \Sigma Y$. It is easy to check directly that $\operatorname{Im} e_{*}$ $\subset \operatorname{Ker}\left[(\Omega \Phi)_{*}-\rho_{*}\right]$ and therefore by the second part of Corollary 3.3 $\operatorname{Im} e_{*}^{*}=\operatorname{Ker}\left[(\Omega \Phi)_{*}-\rho_{*}\right]$. (In fact, the last relation admits also a purely topological proof.) Since $e_{*}$ is a monomorphism, $S\left(H_{*}(\Omega \Sigma Y)\right)=H_{*}(Y)$ as a co-algebra and its dual $S^{*}\left(H_{*}(\Omega \Sigma Y)\right)=\left(H_{*}(Y)\right)^{*}=H^{*}(Y)$ as an algebra.

REMARK 3.4. In the previous analysis we could have replaced the use of a coefficient field by integer coefficients, provided no torsion is present.

REMARK 3.5. In the general case of a co-multiplicative object $(X, \Phi)$ in $\mathscr{T}_{H}$, the original proof of the Bott-Samelson theorem shows that $H_{*}(\Omega X)$ $=T(M)$, where $M \subset H_{*}(\Omega X)$ is a submodule mapped isomorphically onto $H_{*}(X)$ by the homology suspension $\sigma$. We know on the other hand by Theorem 1.1, that also $H_{*}(\Omega X)=T(N)$, where $N$ is the module of normed elements. This means that for any $m \in M, m=n+d$, where $n \in N$ and $d$ is decomposable. Thus $\sigma(m)=\sigma(n)$ and we can always normalize $M$ by choosing $M=N$. This normalization has the advantage that $N$ is natural with respect to maps of co-multiplicative objects, i.e., if $f:\left(X_{1}, \Phi_{1}\right) \rightarrow\left(X_{2}, \Phi_{2}\right)$ then $(\Omega f)_{*}\left(N_{1}\right) \subset N_{2}$.

4. Proof of the key lemmas. Recall that we have denoted by $\mathscr{R}_{n}(\alpha, \beta)$ the set of all alternating sequences on length $n$ formed by using the symbols $\alpha$ and $\beta$. For a fixed $n, \mathscr{R}_{n}(\alpha, \beta)$ consists of exactly two elements: one sequence $(\alpha, \beta, \alpha, \ldots)$ starting with $\alpha$ and another sequence $S_{n}=(\beta, \alpha, \beta, \ldots)$ starting with $\beta$. The sequences $S_{n}, n \geqq 2$, will be called special; $\mathscr{S}(\alpha, \beta)$ will denote the set of all special sequences of any length $(\geqq 2)$.

Let now $\alpha, \beta, \gamma, \cdots$ be any finite set of symobols and let $\mathscr{J}_{n}(\alpha, \beta, \gamma, \cdots)$ denote the set of all sequences of length $n$ formed by using these symbols. A sequence $I \in \mathscr{J}_{n}(\alpha, \beta, \gamma, \ldots)$ is mixed if all the possible symbols occur in $I$. The set of all mixed sequences of length $n$ will be denoted by $\mathscr{M}_{n}(\alpha, \beta, \gamma, \cdots)$. Finally

$$
\begin{aligned}
& \mathscr{J}(\alpha, \beta, \gamma, \cdots)=\bigcup_{n} \mathscr{J}_{n}(\alpha, \beta, \gamma, \cdots), \\
& \mathscr{M}(\alpha, \beta, \gamma, \cdots)=\bigcup_{n} \mathscr{M}_{n}(\alpha, \beta, \gamma, \cdots) .
\end{aligned}
$$

Obviously $\mathscr{R}_{n}(\alpha, \beta) \subset \mathscr{M}_{n}(\alpha, \beta), n \geqq 2$.

Let $N^{\prime}$ and $N^{\prime \prime}$ be two positively graded modules over a fixed commutative ring $\Lambda$ and let $T\left(N^{\prime}\right), T\left(N^{\prime \prime}\right)$ and $T\left(N^{\prime}+N^{\prime \prime}\right)$ denote the tensor algebras of the corresponding modules. We have then the natural isomorphism

$$
\begin{aligned}
& \left\langle T\left(i_{1}\right), T\left(i_{2}\right)\right\rangle: T\left(N^{\prime}\right) * T\left(N^{\prime \prime}\right) \approx T\left(N^{\prime}+N^{\prime \prime}\right), \\
& i_{1}(a)=(a, 0), \quad i_{2}(b)=(0, b), \quad a \in N^{\prime}, \quad b \in N^{\prime \prime},
\end{aligned}
$$


whereby the two sides may be identified. Thus

$$
T\left(N^{\prime}\right) * T\left(N^{\prime \prime}\right)=\Lambda+\Sigma N_{J}, \quad J \in \mathscr{J}\left({ }^{\prime},{ }^{\prime \prime}\right),
$$

where

$$
N_{J}=N^{\jmath_{1}} \otimes \cdots \otimes N^{j_{2}}, \quad J=\left(j_{1}, \cdots, j_{n}\right) .
$$

Let $(A, \Phi)$ be a co-multiplicative object in $\mathscr{A}$ and let $A^{p}$ denote the homogeneous component of $A$ of degree $p$. We now proceed to give an inductive definition of the module $N$ of normed elements. Let us assume that $N \cap A^{1}=A^{1}$ and that $N^{p}=N \cap A^{p}$ has been defined for all $p \leqq k-1$. Let $N_{(k-1)}=N^{1}+\cdots+N^{k-1}$. The imbedding $N_{(k-1)} \subset A$ can be extended to a unique algebra homomorphism

$$
n: T(N) \rightarrow A
$$

(we shall omit the subscript in $N_{(k-1)}$, since this cannot lead to confusion). Our inductive hypothesis is

$$
n \mid T(N)^{p}: T(N)^{p} \approx A^{p}, \quad p \leqq k-1 .
$$

As a consequence of $\left(2.4_{k-1}\right)$ we can identify $\left(A_{I}\right)^{k}, I \in \mathscr{R}\left({ }^{\prime}, "\right)$, with $|I|$ = length $I \geqq 2$, with a sum of form $\left(\sum N_{J}\right)^{k}, J \in \mathscr{M}\left({ }^{\prime}, "\right)$. This yields

$$
(A * A)^{k}=A^{\prime k}+A^{\prime \prime k}+\left(\sum N_{J}\right), \quad J \in \mathscr{M}\left({ }^{\prime},{ }^{\prime \prime}\right)
$$

and correspondingly, for $a \in A^{k}$

$$
\Phi(a)=a^{\prime}+a^{\prime \prime}+\sum \Phi_{J}(a) \text {, where } \Phi_{J}(a) \in N_{J}, \quad J \in \mathscr{M}\left({ }^{\prime}, "{ }^{\prime \prime}\right) .
$$

We shall always write $\Phi_{r}$ instead of $\Phi_{S_{r}}$, where $S_{r}$ is the unique special sequence of length $r$ formed from the symbols " and ".

DEFinition $N_{k}$. An element $a \in A^{\prime}$ is normed if in (4.04), $\Phi_{s}(a)=0$ for all $s \geqq 2$.

The tensor algebra $T(N)$ is bigraded $T(N)=\sum F^{p k}$, where the first degree of $n_{1} \otimes \cdots \otimes n_{p}$ is $p$ and its second degree is $\operatorname{deg} n_{1}+\cdots+\operatorname{deg} n_{p}$. $T(N) * T(N)=T\left(N^{\prime}+N^{\prime \prime}\right)=\sum N_{J}$ is also bigraded by $\sum G^{p k}$. Let $\nabla$ $=\langle 1,1\rangle: T\left(N^{\prime}\right) * T\left(N^{\prime \prime}\right) \rightarrow T(N)$ be the "folding" map. The following properties are easily checked

$$
\begin{gathered}
\Phi_{s}\left(A^{k}\right) \subset G^{s k} ; \\
\Phi_{s}\left(n\left(F^{p k}\right)\right) \subset \sum_{q \geq p} G^{q k} ; \\
\nabla\left(G^{p k}\right) \subset F^{p k} ;
\end{gathered}
$$

$\nabla$ is a monomorphism when restricted to any $N_{J}$.

By (4.1) and (4.2) we have

$$
\Phi_{s}\left(n\left(F^{p k}\right)\right)=0 \quad \text { if } s<p ;
$$

moreover, it follows easily from the definition of $\Phi$ that 


$$
\nabla \Phi_{s}(n(a))=a \quad \text { if } a \in F^{s k} .
$$

Proof of Proposition 2.3. (i) $n: T(N)^{k} \rightarrow A^{k}$ is onto. Let $a \in A^{k}$; set $a_{1}=a, a_{s}=a_{s-1}-n \nabla \Phi_{s}\left(a_{s-1}\right), s \geqq 2$. By (4.1) and (4.3) we have

$$
\nabla \Phi_{s}\left(a_{j}\right) \subset F^{s k}
$$

therefore, by (4.5),

$$
\Phi_{m}\left(n \nabla \Phi_{s}\left(a_{s-1}\right)\right)=0 \quad \text { and thus } \Phi_{m}\left(a_{s}\right)=\Phi_{m}\left(a_{s-1}\right) \quad \text { for } m<s .
$$

Let us assume as an induction hypothesis that $\Phi_{m}\left(a_{s-1}\right)=0$ for all $m \leqq s-1$; it follows that from (4.8) then $\Phi_{m}\left(a_{s}\right)=0$ for all $m<s$. But according to (4.7) and to (4.6)

$$
\nabla \Phi_{s}\left(a_{s}\right)=\nabla \Phi_{s}\left(a_{s-1}\right)-\nabla \Phi_{s} n \nabla \Phi_{s}\left(a_{s-1}\right)=0 .
$$

By 4.4 this proves in particular that

$$
\Phi_{m}\left(a_{k}\right)=0 \text { for all } m \leqq k ;
$$

since on the other hand $G^{m k}=0$ for $m>k$ it follows from (4.1) that $a_{k}$ is normed. This completes the proof of (i) since $a-a_{k}$ lies in the image of $n$ by construction.

(ii) $n: T(N)^{k} \rightarrow A^{k}$ is a monomorphism. Let $a \in \operatorname{ker} n, a=a^{1}+\cdots+a^{k}$, $a^{j} \in F^{j k}$. Obviously $\Phi_{s}\left(n\left(a^{1}\right)\right)=\Phi_{s}\left(a^{1}\right)=0$ for $s \geqq 2$ (since $F^{1 k}=N^{k}$ ). By (4.5) and (4.6), $a^{2}=\nabla \Phi_{2} n\left(a^{2}\right)=\nabla \Phi_{2} n(a)=0$. In the same way, by applying successively $\nabla \Phi_{3}, \nabla \Phi_{4}$, we prove that $a^{s}=0$ for all $s \geqq 2$ and therefore $a=a^{1} \in N^{k}$. Since $n \mid N^{k}$ is the identity, $n(a)=0$ implies $a=0$.

In order to prove Lemma 2.5 we shall use the notation described at the beginning of this section. By definition, $\mathscr{N}_{n}(1,2,3)=\mathscr{M}_{n}(1,2,3)$ for $n \geqq 4$ and $\mathscr{N}_{3}(1,2,3)=\mathscr{M}_{3}(1,2,3)-\{(1,2,3)\}$ (we exclude the increasing sequence $(1,2,3))$. Let us define the maps

$$
\begin{aligned}
& \phi: \mathscr{N}_{n}(1,2,3) \rightarrow \mathscr{N}_{n}(1,2), \\
& \psi: \mathscr{N}_{n}(1,2,3) \rightarrow \mathscr{N}_{n}(1,2)
\end{aligned}
$$

by

$$
\begin{array}{ll}
\phi\left(\left(i_{1}, \cdots, i_{n}\right)\right)=\left(j_{1}, \cdots, j_{n}\right), & j_{k}=\max \left(i_{k-1}, 1\right), \\
\psi\left(\left(i_{1}, \cdots, i_{n}\right)\right)=\left(j_{1}, \cdots, j_{n}\right), & j_{k}=\min \left(i_{k}, 2\right) .
\end{array}
$$

Consider the weakest equivalence relation on $\mathscr{M}_{n}(1,2)$ compatible with the relation $K_{1} \sim K_{2}$ if $K_{1}=\phi(I), K_{2}=\psi(I)$ for the same $I \in \mathscr{N}_{n}(1,2,3)$.

Lemma 4.1. All the elements of $\mathscr{M}_{n}(1,2)$ are equivalent under the above relation.

Proof. For $n=3$ the proof is obtained by direct verification. If $n>3$, let $K=\left(k_{1}, \cdots, k_{n}\right), M=\left(m_{1}, \cdots, m_{n}\right), k_{i}, m_{i}=1$ or 2 . If $k_{i} \leqq m_{i}$ for all $i$, 
then $K \sim M$; indeed $L=\left(l_{1}, \cdots, l_{k}\right)$, where $l_{i}=k_{i}+m_{i}-1$ satisfies $\phi(L)$ $=K, \psi(L)=M$.

(i) If for some $i, k_{i}=m_{i}=1$, set $l_{s}=\max \left(k_{s}, m_{s}\right)$. Then $L=\left(l_{1}, \ldots, l_{n}\right)$ $\in \mathscr{M}_{n}(1,2)$ and by the previous remark $K \sim L \sim M$. If for some $i, k_{i}$ $=m_{i}=2$, set $l_{s}=\min \left(k_{s}, m_{s}\right)$ and again $K \sim L \sim M$.

(ii) Assume now that for all $i, k_{i} \neq m_{i}$. Since $n \geqq 3$ we may assume that one of the sequences $K$ or $M$, say $K$, contains the symbol 1 at least twice. By replacing one of these 1's by 2 we get another sequence $K^{\prime}$ such that $K \sim K^{\prime}$ and we are able to apply (i) to $K^{\prime}$ and $M$.

Lemma 2.5 will be easily deduced from the following

Lemma 4.2. If $(A, \Phi)$ is an associative c.-m. object in $A$ and $N$ is the module of all normed elements then any $a \in N$ is semi-primitive, i.e, $\Phi(a)=a^{\prime}+a^{\prime \prime}$ $+\sum a_{1}^{\prime} a_{j}^{\prime \prime}$, where $a_{i}^{\prime} \in N^{\prime}, a_{j}^{\prime \prime} \in N^{\prime \prime}$.

Proof of Lemma 4.2. According to Lemma 2.4, $A=T(N)$ and therefore by (4.1)

$$
\begin{aligned}
A * A & =T\left(N^{\prime}\right)+T\left(N^{\prime \prime}\right)+\sum N_{J}, \quad J \in \mathscr{M}(1,2), \\
A * A * A & =T\left(N^{\prime}\right)+T\left(N^{\prime \prime}\right)+T\left(N^{\prime \prime \prime}\right)+\sum N_{K}+\sum N_{L},
\end{aligned}
$$

where $K \in \mathscr{M}(1,2) \cup \mathscr{M}(1,3) \cup \mathscr{M}(2,3)$ and $L \in \mathscr{M}(1,2,3)$ (for technical reasons we write 1 for ', 2 for " and 3 for "').

Let $a \in N$. Then

$$
\begin{aligned}
& (\Phi * 1) \Phi(a)=a^{\prime}+a^{\prime \prime}+a^{\prime \prime \prime}+\sum f_{K}(a)+\sum f_{L}(a), \\
& (1 * \Phi) \Phi(a)=a^{\prime}+a^{\prime \prime}+a^{\prime \prime \prime}+\sum g_{K}(a)+\sum g_{L}(a),
\end{aligned}
$$

where $f_{K}(a), g_{K}(a) \in N_{K}, f_{L}(a), g_{L}(a) \in N_{L}$. By associativity of $\Phi$ we must have

$$
f_{L}(a)=g_{L}(a), \quad L \in \mathscr{M}(1,2,3) .
$$

Lemma 4.2 will be proved by proving for all $k$ and $s, s \leqq k$, the following statement

$$
\Phi_{J}(a)=0, \quad J \in \mathscr{M}_{n}(1,2), \quad J \neq(1,2)
$$

for $a \in N^{p}, p \leqq k-1$ and all $n \geqq 2$ and for $a \in N^{k}, 2 \leqq n \leqq s$.

Since for any $k,(4.2 . k-1 . k-1)$ and (4.2.k.s), $s=1,2$, are clearly equivalent, it will be enough to use induction on $s$. (4.2.1.1) being trivial we shall assume (4.2.k.s - 1), $s \geqq 3$ and prove (4.2.k.s.).

First we shall notice that any $b \in\left(N_{J}\right)^{k}, J \in \mathscr{M}(1,2)$ is a sum of products of elements lying in $N^{p_{i}}$ or $N^{\prime \prime p_{i}}$ with all $p_{i} \leqq k-1$. This enables us by $(4.2 . k . s-1)$ to assume in computing $(\Phi * 1)(b)$ and $(1 * \Phi)(b)$, that

$$
\Phi(a)=a^{\prime}+a^{\prime \prime}+\sum a_{i}^{\prime} a_{j}^{\prime \prime} .
$$

On the other hand it follows from the definition of $\Phi$ and that of the 
maps $\phi$ and $\psi$ in (4.10) that if the sequences $J$ and $L$ have the same length, i.e., $|J|=|L|$ and $J \in \mathscr{M}(1,2), L \in \mathscr{M}(1,2,3)$,

$$
\begin{gathered}
(\Phi * 1)_{L}\left(N_{J}\right)=0 \quad \text { if } J \neq \phi(L), \quad|J|=|L|, \\
(\Phi * 1)_{L} \mid N_{\phi(L)}: N_{\phi(L)} \approx N_{L},
\end{gathered}
$$

and analogously

$$
\begin{gathered}
(1 * \Phi)_{L}\left(N_{J}\right)=0 \quad \text { if } J \neq \psi(L), \quad|J|=|L|, \\
(1 * \Phi)_{L} \mid N_{\psi(L)}: N_{\psi(L)} \approx N_{L} .
\end{gathered}
$$

By (4.12) we have for any $a \in N^{k}$

$$
\begin{aligned}
& (\Phi * 1) \Phi_{(1,2)}(a)=\Phi_{(1,3)}(a)+\Phi_{(2,3)}(a)+\Phi_{(1,2,3)}(a), \\
& (1 * \Phi) \Phi_{(1,2)}(a)=\Phi_{(1,2)}(a)+\Phi_{(1,3)}(a)+\Phi_{(1,2,3)}(a) .
\end{aligned}
$$

Now the remark that by $(4.2 . k . s-1)$ the only $\Phi_{J}(a)$, with $|J|<s$ which can be $\neq 0$ for $a \in N^{k}$ is $\Phi_{(1,2)}(a)$, yields together with (4.14) and the first lines of (4.13) and $\left(4.13^{\prime}\right)$

$$
\begin{aligned}
& f_{L}(a)=(\Phi * 1)_{L} \Phi_{\phi(L)}(a), \\
& g_{L}(a)=(1 * \Phi)_{L} \Phi_{\psi(L)}(a), \quad L \in \mathscr{N}(1,2,3)
\end{aligned}
$$

(where $\mathscr{N}(1,2,3)$ is the set of all mixed sequences with the exception of $(1,2,3))$. By using now (4.11) and the second lines of (4.13) and (4.13') and by referring to the definition of equivalence of sequences used in Lemma 4.1, we get that $\Phi_{J}(a)=0$ implies that $\Phi_{K}(a)=0$ if $J \sim K$. But, by Lemma 4.1 all sequences in $\mathscr{M}_{s}(1,2)$ are equivalent and by definition of normed elements, at least for one sequence $J \in \mathscr{M}_{s}(1,2)$, we have $\Phi_{J}(a)=0$; thus $\Phi_{J}(a)=0$ for all $J$. This completes the proof of (4.2.k.s.) and of Lemma 4.2.

Proof of Lemma 2.5. According to Lemma 4.2, if $a \in N, \Phi(a)$ has the form (4.12) with $a_{i}^{\prime} \in N^{\prime}, a_{j}^{\prime \prime} \in N^{\prime \prime}$. An easy computation based on (2.5) shows that $\Phi(a)=\rho(a)$ (see (2.6) and $\left(2.6^{\prime}\right)$ ) and thus $a \in S$. The second part of Lemma 2.5 is an immediate consequence of the fact that $N$ is a coalgebra.

\section{REFERENCES}

1. R. Bott and H. Samelson, On the Pontryagin product in spaces of paths, Comment. Math. Helv. 27 (1953), 320-337.

2. B. Eckmann and P. J. Hilton, Group-like structures in general categories. I, Math. Ann. 145 (1962), 227-255; II, ibid. 151 (1963), 150-186; III, ibid. 150 (1963), 165-187.

3. Structure maps in group theory, Fund. Math. 50 (1961/62), 207-221.

4. D. M. Kan, On monoids and their dual, Bol. Soc. Mat. Mexicana (2) 3 (1958), 52-61.

5. __ A combinatorial definition of homotopy groups, Ann. of Math (2) 67 (1958), 282-312.

CORNELL UNIVERSITY,

ITHACA, NEW YORK 